\title{
La especialidad en Organización y Administración de Salud Pública y la Escuela Cubana de Salud Pública
}

\author{
The public health organization and management specialty and \\ the National School of Public Health
}

\author{
Dr. Luis Suárez Rosas \\ Escuela Nacional de Salud Pública. La Habana, Cuba.
}

\begin{abstract}
RESUMEN
Se realiza un recorrido histórico que abarca el desarrollo de la Escuela Cubana de Salud Pública, pasando por la etapa de la Escuela de Higienistas Cubanos con el Dr. Carlos J. Finlay al frente, hasta llegar al momento actual. Se destaca la negativa repercusión de la extinción de la especialidad de Organización y Administración de Salud Pública dentro de los estudios de posgrado en Cuba desde hace más de 20 años. Se hace hincapié en que hoy más que nunca en el Sistema Nacional de Salud de Cuba resulta imposible ignorar la necesidad de la existencia de la especialidad, surgida en la práctica y desarrollada desde hace más de 200 años, que si bien se ha llamado de diversos modos en el mundo, ha sido y es el diario quehacer de un sin número de profesionales que intentan dirigir sobre bases científicas las tareas propias de los sistemas de salud. Disponer de profesionales de la salud pública competentes es una prioridad absoluta para el buen desempeño y perfeccionamiento constante del Sistema Nacional de Salud y que en la consecución de este noble propósito, la especialidad de Organización y Administración de Salud Pública, una vez que surja de nuevo con este nombre o con otro, se consolide en el tiempo y cumpla su encargo social coherentemente, podrá ser de suma utilidad.
\end{abstract}

Palabras clave: Escuela Cubana de Salud Pública, Sistema Nacional de Salud, educación de posgrado, especialidad de Organización y Administración de Salud Pública, Cuba. 


\section{ABSTRACT}

A historical review covering the development of the Cuban School of Public Health, the stage of the Cuban School of Hygienists led by Dr. Carlos J. Finlay, and the present time was made. Emphasis was made on the negative impact of the elimination of the public health organization and management specialty from the graduate studies in Cuba for more than 20 years. It is stressed that today more than ever, it is not longer possible to ignore the need for this over 200 years-old specialty to be rescued; even though it has been called differently worldwide, it has been and it is the daily work of a number of professionals who attempt to lead the tasks inherent to the health systems on a scientific basis. The availability of competent professionals in public health is a priority for good performance and constant improvement of the national health systems, and to accomplish this, the public health organization and management specialty could be very useful, once it re-emerges under the same or a new name, becomes stronger and coherently fulfils its social task.

Key words: Cuban School of Public Health, national health system, post graduate education, public health organization and management specialty.

\section{NTRODUCCI ÓN}

En el transcurso del curso académico 1989-1990 las autoridades del Ministerio de Salud Pública de Cuba decidieron que no se aceptaran en lo adelante nuevos ingresos para la especialidad de Organización y Administración de Salud Pública en las dos variantes que estaban vigentes en aquel momento: la Residencia a Tiempo Completo y la Residencia por Encuentros. La dirección de la Facultad de Salud Pública del entonces Instituto Superior de Ciencias Médicas de La Habana, institución donde se cursaba la especialidad en cuestión, tratando de preservar tan significativos estudios de posgrado, presentó a partir de ese momento histórico concreto dos nuevas propuestas de planes de estudio de la Residencia elaborada por los más calificados profesores de la esfera de la Salud Pública de entonces y otros que sin pertenecer a la especialidad son en la actualidad considerados paradigmas de la docencia de las Ciencias Médicas en Cuba. En el archivo personal del autor consta el documento "Especialidad en Organización y Administración de Salud Pública. Área de Docencia. Ministerio de Salud Pública. La Habana. Octubre 4 de 1991" donde aparece la segunda propuesta presentada el 4 de Octubre de 1991 y que plantea lo siguiente:

Preparar un especialista de alto nivel científico técnico en el campo de la Salud Pública, como una segunda especialidad a partir del especialista de Medicina General Integral o de otra de las especialidades médicas o estomatológicas. Reiniciar la residencia en dos años y medio a tiempo completo, que tenga un primer año con las disciplinas generales, como el resto de las especialidades de Salud Pública (Bioestadística e Higiene y Epidemiología). El programa de la residencia hará énfasis en una fuerte base teórica y práctica, estableciendo las habilidades reales y prioritarias del desarrollo de la Salud Pública. 
Más de 20 años después de estos hechos, la especialidad de Organización y Administración de Salud Pública continúa sin estar entre los estudios de posgrado que se realizan en el país.

¿Qué ha sucedido durante los años de ausencia de los procesos de formación de especialistas en Organización y Administración de Salud Pública?

En la década de los 90, la existencia del Sistema Único de Preparación y Superación de los Cuadros del Estado y sus Reservas (SUPSCER), propició que se desarrollaran cursos con un máximo de duración de cuatro semanas que funcionaron en la propia Facultad de Salud Pública, para la capacitación a directores con un carácter selectivo y con el objetivo de que los cursos se replicaran a nivel de provincia y de municipios. En estos cursos se tenían en cuenta las potencialidades de los directivos según la entidad que dirigían y sus características y se aplicaba un acercamiento a la determinación de necesidades de aprendizaje en función de las temáticas seleccionadas y el tipo de institución del Sistema Nacional de Salud, esto es, hospitales, clínicas estomatológicas y policlínicos, fundamentalmente. Estos cursos sirvieron, entre otras cosas, para mantener con vida docente e investigativa a la Facultad de Salud Pública, conjuntamente con la existencia de la Maestría Internacional de Salud Pública y la impartición de la especialidad de Bioestadística en la institución (con anterioridad las especialidades de Higiene y de Epidemiología habían pasado para el Instituto Nacional de Higiene, Epidemiología y Microbiología), pero la Especialidad de Organización y Administración de Salud Pública continuaba sin aparecer en escena.

Más adelante, al extinguirse el SUPSCER y fusionarse el Centro Nacional de Perfeccionamiento Médico y la Facultad de Salud Pública de la hoy Universidad de Ciencias Médicas de La Habana, se crea la Escuela Nacional de Salud Pública (ENSAP) y con ella el diseño de Cursos de Dirección dirigidos a los directivos del Sistema Nacional de Salud. Estos Cursos para Directivos se han perfeccionado con el decursar del tiempo mediante nuevos diseños dentro de la figura de los Diplomados, acorde a la Resolución Ministerial del MES No. 6 del año 1996, que los describe como una figura organizativa del posgrado vinculada a la necesidad de dar respuestas en un tiempo relativamente corto a determinadas necesidades preservando la especificidad relacionada con el perfil de desempeño requerido que le da origen y los modos de actuación necesarios para el abordaje de determinadas funciones laborales de carácter profesional.

Igualmente, con la ENSAP se incrementa el proceso de formación doctoral en Ciencias de la Salud y se impulsa la realización de maestrías en el campo de la salud pero todo ello ha ocurrido sin que aún aparezca una nueva modalidad de la especialidad de Organización y Administración de Salud Pública, el eslabón faltante de la cadena del posgrado en el campo de la Salud Pública, que va dirigida, como las especialidades médicas y estomatológicas en general, a garantizar la calidad del desempeño profesional y su repercusión en la sociedad. En el año 1995 se autoriza por el Ministerio de Educación Superior en Cuba la realización de maestrías dirigidas a profesionales nacionales, (iniciada con el surgimiento del Instituto de Desarrollo de la Salud en 1976 con la categoría de Maestría Internacional la de Salud Pública que adquirió un renombrado prestigio con una demanda significativa de profesionales procedentes de diferentes países y contextos sociales aspirando a cursarla). Por esta han pasado numerosos profesionales de la salud del país. De alguna manera, la Maestría de Salud Pública (y otras maestrías afines surgidas también en estas dos últimas décadas), con el propósito más general de proporcionar a los educandos el conocimiento de los fundamentos históricos, filosóficos, éticos, políticos y económicos de la salud pública, así como de sus 
métodos de dirección e investigación, contribuyó a mantener viva la llama del pensamiento sanitario en la esfera del posgrado y en el país.

No puede dejarse de tomar en consideración que tantos años sin formación de especialistas en Organización y Administración de Salud Pública ha dejado una huella y un costo que se traducen en un daño social que es necesario revertir lo antes posible atendiendo a que el principal problema del Sistema Nacional de Salud, se genera por ser "un sistema complejo dada la presencia de un número elevado de elementos, relaciones, propiedades, jerarquías y fronteras que tienen numerosas formas de combinarse y recombinarse y que están sometidos a una dinámica muy elevada", ${ }^{1}$ lo que afecta su capacidad de dirección en sus diferentes niveles y por ende su capacidad resolutiva, sostenible, perdurable, adaptable a los cambios y a las variaciones de su contexto y sus circunstancias.

La realidad es que después de haber transcurrido prácticamente el paso de una generación, la especialidad de Organización y Administración de Salud Pública sigue sin estar dentro de los estudios de posgrado que se realizan en el espectro de especialidades de las Ciencias Médicas a pesar de algunos esfuerzos realizados en los últimos tiempos para reiniciar la formación de especialistas.

El sociólogo alemán Karl Mannheim realizó en 1928 contribuciones importantes en la sociología del conocimiento con su concepto de generación, que no como hasta entonces comprendía los 30 años, sino que la caracteriza por acontecimientos generacionales, es decir, hechos que marcan la niñez y la juventud y que tendrán influencia el resto de la vida. ${ }^{2}$

La vigésima segunda edición del Diccionario de la Lengua Española, Madrid 2001, define la palabra generación como el "conjunto de personas que por haber nacido en fechas próximas y recibido educación e influjos culturales y sociales semejantes, se comportan de manera afín o comparable en algunos sentidos."

De cualquier manera, una generación de profesionales de la salud de Cuba, se ha formado sin que tuvieran la opción de cursar estudios de posgrado dentro de la especialidad de Organización y Administración de Salud Pública como especialidad propiamente dicha. He ahí, un daño social analizado desde una visión antropológica.

\section{LAS ESPECI ALI DADES EN EL SISTEMA NACIONAL DE SALUD DE CUBA}

La especialidad es una forma académica de posgrado que en el Sistema Nacional de Salud de Cuba ha estado siempre caracterizada por un alto rigor en la actualización y profundización de los conocimientos de la rama en cuestión y la formación de habilidades más específicas para desempeñar los modos de actuación descritos en el modelo del especialista. Es una respuesta de la Salud Pública cubana a las necesidades surgidas de su propio desarrollo. El especialista, además, profundiza en los métodos de investigación para elevar la efectividad de su labor y garantizar la producción de nuevos conocimientos, en función de garantizar los niveles de calidad como prestador de servicios de salud.

La Educación de Posgrado, se considera el nivel superior del Sistema Nacional de Educación o cuarto nivel, surge como una necesidad imprescindible para mejorar los indicadores de desarrollo del país, a partir del resultado de procesos avanzados 
de investigación en función de las necesidades sociales, económicas y culturales de la población, así como a los requerimientos tecnológicos del aparato productivo y al intercambio internacional, está dirigida a los egresados de la Educación Profesional y responde además a los intereses individuales de los profesionales. ${ }^{3}$

El posgrado se organiza en dos direcciones fundamentales: La Formación Académica del Posgrado (especializaciones, maestrías y doctorados) y la Superación Continua de los egresados universitarios durante su vida profesional (adiestramiento laboral, complementación para determinados puestos de trabajo, reorientación, actualización, profundización).

Los objetivos de la formación académica de posgrado están encaminados a ofrecer al profesional un nivel cualitativamente superior desde el punto de vista profesional y científico: la especialización de posgrado proporciona a los graduados universitarios la profundización y ampliación de los conocimientos en áreas particulares de la profesión. ${ }^{4}$

En el propio Sistema Nacional de Salud se forman los recursos humanos (médicos y paramédicos) así como la formación académica y superación de todos los profesionales que laboran en el mismo.

\section{Antecedentes históricos de la especialidad de Organización y Administración de Salud Pública}

La especialidad, como práctica diferenciada de médicos y otros profesionales, existe desde hace más de dos siglos, período en que ha recibido distintos nombres (de policía médica a medicina colectiva, con más frecuencia administración de salud, medicina social y predominantemente salud pública), lo que ha sido reflejo de concepciones diferentes pero que tienen más de común que de contradictorio.

La especialidad surgió y se desarrolló en cada lugar a partir de necesidades sociales del desarrollo de la protección de la salud de la población, posee un objeto de estudio y amplio contenido de trabajo, utiliza métodos de diversas ciencias, es materia de enseñanza, posee un amplio campo de investigaciones científica y ha alcanzado a nivel internacional un importante lugar en su organización social y laboral.

La existencia formal institucionalizada en esta especialidad es también de larga historia. Existió ya en 1685 un Colegio de Sanitarios en Prusia, pero como es conocido hoy, es en 1920 cuando se crean las instituciones docentes modernas en la propia Alemania, una de ellas, como cátedra universitaria. En los Estados Unidos de América en 1910 se expidió por la Universidad de Michigan el primer título de Especialista en Salud Pública. En América Latina, las Escuelas de Salud Pública de México y de Sao Paulo, Brasil, surgen en el primer cuarto de siglo. En Cuba, se inician los esfuerzos por desarrollar esta enseñanza especializada de posgrado al crearse por Decreto Presidencial de 17 de Enero de 1927 el Instituto "Finlay" como centro rector de la Escuela Sanitaria Nacional, institución para la formación del personal sanitario, a partir de los acuerdos tomados en la v Conferencia Panamericana de la Salud. Esta propuesta fue formulada por el Dr. Juan Guiteras Gener en 1911 pero ello no se logra hasta el año 1927 como señalamos anteriormente. La gestión del Dr. Guiteras duró, como podemos observar, 16 años en concretarse. La historia de esta Escuela estuvo marcada por la falta de apoyo gubernamental, escasez de recursos y cambios en su dependencia que siempre excluyeron su integración a la Universidad de La Habana. Al final de la República Burguesa se ocupaba de la formación de algunos técnicos medios. 
Las necesidades del desarrollo de la salud pública y de la formación de especialistas en esta rama del saber se tornaron evidentes desde los primeros años de la Revolución. Así, en 1961 ya se había organizado un curso de salud pública de seis meses de duración para los funcionarios y especialistas que asumían responsabilidades en las recién creadas Direcciones Regionales de Salud Pública. A ello siguió la organización de una primera Escuela de Salud Pública donde el curso se extendió a un año académico. Importante colaboración se recibió en esta etapa de docentes latinoamericanos de las Escuelas de Salud Pública de Chile y México, fundamentalmente. Tuvo un progresivo fortalecimiento hasta el momento en que se descentralizó provocando que en realidad su claustro se dispersara al igual que sus recursos materiales (Perspectiva de la Especialidad de Organización y Administración de Salud Pública. Área de Docencia. Ministerio de Salud Pública. La Habana. Octubre 4 de 1991. Archivo personal de autor).

A mediados de la década de los años 70 se tomó de nuevo conciencia de la necesidad de su resurgir y es entonces cuando el área de docencia del Ministerio de Salud Pública organiza un curso con un año de duración. Esta área se integró en 1976 al Instituto de Desarrollo de la Salud (IDS) fundado en ese mismo año. Los alumnos que se encontraban cursando el curso de Administración de Salud en la Unidad Docente Nacional "Carlos J. Finlay", sede del curso, se trasladaron al IDS ya en calidad de residentes de la especialidad de Organización y Administración de Salud Pública. En el IDS se alcanzó una estabilidad en la formación de especialistas en la mencionada especialidad al igual que en las de Bioestadística, Epidemiología e Higiene, hasta que estas dos últimas pasaran a la sede del Instituto Nacional de Higiene, Epidemiología y Microbiología (INHEM).

El plan de formación de especialistas en Organización y Administración de Salud sufrió constantes cambios y así en 1983 se abre la modalidad de cursos por encuentros. Esta última etapa estuvo marcada por la carencia de una política estable en relación con la especialidad, lo que se agravó con la extinción en el año 1986 del IDS, institución que dio paso a la Facultad de Salud Pública subordinada al entonces Instituto Superior de Ciencias Médicas de La Habana.

Todas estas venturas y desventuras, ir y venir de la especialidad y sus instituciones sedes, contrasta de modo notable con el real desarrollo de la salud pública en sus realizaciones práctica e indicadores alcanzados en el país.

Están frescas en mis recuerdos y en mis oídos las palabras del ya fallecido Profesor estadounidense Milton Terris, uno de los más sobresalientes especialistas en Salud Pública de nuestra contemporaneidad, cuando en una visita suya a la sede de la Facultad de Salud Pública en el año 1990 decía que "Cuba podría tener la mejor Escuela de Salud Pública del mundo."

El Profesor Milton Terris no hacía esa afirmación por complacencia. Nada más lejano de su forma de pensar y actuar pues conocía perfectamente el desarrollo de la salud pública en Cuba y el prestigio de sus paradigmas a lo largo de los años.

Al profundizar el análisis histórico de esta temática encontramos que los antecedentes en Cuba de la enseñanza de la salud pública se remontan al año 1842 cuando como parte de la Reforma Universitaria se crea la cátedra de Policía Médica e Higiene Privada y Pública en la Facultad de Medicina. Más tarde se reduce a la asignatura de Higiene y Legislación Sanitaria en contraposición con el desarrollo que alcanza la salud pública cubana a partir de 1902 cuando Carlos J. Finlay se convierte en su principal gestor y se crea el 28 de enero de 1909 la primera Secretaría de Salud del mundo. Previamente, con Finlay como figura central había 
surgido la Escuela de Higienistas Cubanos integrada por un grupo de prestigiosos higienistas de la época.

Tras constantes amenazas durante tres décadas, la Escuela cierra sus puertas en los años 50 pero logra reactivarse al triunfo revolucionario en 1959 a través de la Ley No. 207 que regula el funcionamiento de la carrera sanitaria. En la etapa revolucionaria se identifican cinco modelos fundamentales:

- 1960-1969 Escuela de Salud Pública.

- 1970-1971 Cerrada.

- 1971-1975 Unidad Docente Carlos J. Finlay.

- 1976-1986 Instituto de Desarrollo de la Salud.

- 1986- 2000 Facultad de Salud Pública.

- 2000 Escuela Nacional de Salud Pública.

\section{LA ESCUELA CUBANA DE SALUD PÚBLI CA Y SUS BASES FUNDACI ONALES EN LA ESCUELA DE HI GI ENI STAS CUBANOS}

El arribo de Cristóbal Colón con sus tres carabelas el 27 de Octubre de 1492 a Cuba, permitió a los españoles iniciar desde los primero años del siglo xvı la conquista del país. No es hasta 1634 que se funda la primera organización de la salud pública cubana, el Real Tribunal del Protomedicato de La Habana, que era a su vez la primera organización de la salud pública española, la cual había sido mandada a instaurar en América por Ley de Indias de 1570 y que con anterioridad a Cuba se había fundado solamente en los virreinatos de México y Perú. ${ }^{5}$

Desde los primeros años de la colonización había hecho su entrada en Cuba (1520) la viruela, como primera gran enfermedad epidémica y en 1649 la fiebre amarilla, para constituir los dos grandes problemas epidemiológicos que frenaban el desarrollo económico y social del país.

Durante el siglo XIX se producen varios hechos de importancia histórica, relacionados con la Salud Pública en Cuba: la introducción de la vacuna antivariólica por Tomás Romay (1805), la fundación del Cementerio de Espada (1805), la existencia de 25 hospitales en 1850 y la exposición por Carlos J. Finlay en 1881, de su teoría acerca del Culex mosquito, hoy Aedes aegypti como posible agente transmisor de la fiebre amarilla.

La llegada del cólera en 1833, como tercer gran problema del cuadro epidemiológico de Cuba, determina el cierre del ya obsoleto Real Tribunal del Protomedicato de La Habana y su sustitución por otras dos organizaciones de salud, la Junta Superior Gubernativa de Medicina y Cirugía y la J unta Superior Gubernativa de Farmacia, las que solamente duraron diez años y cuyas funciones quedaron incorporadas a las Juntas de Sanidad.

En 1898, concluida la Guerra de Independencia contra la metrópoli española organizada y dirigida hasta su caída en combate el 19 de Mayo de 1895 por José Martí, "el misterio que nos acompaña" al decir del insigne escritor cubano J osé Lezama Lima, se produce la primera ocupación norteamericana en Cuba (18981902). Paradójicamente, este hecho propició una importante labor de higienización a lo largo de todo el país; se tomaron enérgicas medidas contra el muermo, la tuberculosis humana y del ganado, la fiebre tifoidea, viruela, fiebre de borras, paludismo, escarlatina, lepra y otras enfermedades. Se logró reorganizar la salud 
pública con la participación de los médicos del país, pero el hecho de mayor importancia lo constituyó, sin lugar a dudas, la aplicación del descubrimiento de Carlos J. Finlay para la erradicación de la fiebre amarilla, uno de los momentos cumbres de la historia de la salud pública mundial que, sin embargo, se vio enlodado por el más bochornoso intento de plagio que se recuerda en la historia de la medicina. ${ }^{6}$

Al quedar instaurada la República de Cuba el 20 de mayo de 1902, fue nombrado Secretario de Gobernación, Diego Tamayo Figueredo, una de las más importantes figuras de la medicina cubana y como los servicios de sanidad y beneficencia eran dependencias de su Secretaría (Ministerio), nombró al frente de la dirección nacional de los primeros a Carlos J. Finlay y de la dirección nacional de los segundos al ilustre higienista Manuel Delfín Zamora.

Finlay desde el comienzo de su mandato al frente de lo que ha dado en llamarse Escuela de Higienistas Cubanos como se señaló más arriba, trató de estructurar un Sistema Nacional Estatal de Salud que abarcara todas las instituciones de la salud pública del país, ya pertenecieran a la sanidad terrestre o marítima, que entonces dependían de distintas Secretarías o como organismos estatales autónomos.

Los higienistas cubanos, bajo la dirección de Finlay (1902-1908), logran erradicar la fiebre amarilla definitivamente de Cuba (1908), disminuir la mortalidad por tétanos infantil a partir de 1903, establecer de manera permanente la vacunación contra la viruela y elaborar una avanzada legislación en materia sanitaria.

La Escuela de Higienistas Cubanos, entre cuyas grandes figuras no podemos dejar de mencionar, además de Finlay, a Juan Guiteras Gener, Enrique B. Barnet Roque de Escobar, Antonio Díaz-Albertini Mojarrieta, José A. López del Valle Valdés, Arístides Agramonte Simoni, Hugo Roberts Fernández, Jorge Le Roy Cassá, Honoré Lainé Garesche, J oaquín L. Dueñas Pinto, Mario García-Lebredo Arango, J oaquín L. J acobsen Cantos y Emilio Martínez Martínez, va a lograr durante la segunda ocupación militar norteamericana (1906-1909) que las organizaciones sanitarias y de beneficencia sean elevadas a categoría de departamentos nacionales. ${ }^{7}$

Este importante paso permitió que al discutirse una nueva ley sobre la estructura del poder ejecutivo en la Comisión Consultiva, organismo legislativo que había sustituido al Congreso de la República en dicha etapa de ocupación extranjera, se aprobara una Secretaría de Sanidad y Beneficencia, que reunía con categoría ministerial a los Departamentos Nacionales de Sanidad y Beneficencia.

El 28 de enero de 1909, al restablecerse la República y ponerse en vigor la nueva Ley Orgánica del Poder Ejecutivo, entra en funciones la Secretaría de Sanidad y Beneficencia, primer ministerio de salud pública del mundo, cuya creación respondía exclusivamente al desarrollo histórico de la salud pública en el país y a las ideas avanzadas en materia salubristas de la Escuela de Higienistas Cubanos de principios de siglo. ${ }^{8}$

De manera que Cuba fue el primer país del mundo que contó con una Secretaría de Sanidad, cuando en 1909 se convirtió en tal el Departamento Nacional de Sanidad, que desde 1902 estaba adscrito al entonces Ministerio de Gobernación. Tres décadas después, dicho organismo se tituló Ministerio de Salubridad y Asistencia Social, por disposición de la Constitución de 1940. En 1959 su nombre se volvió a modificar por el de Ministerio de Salubridad y Asistencia Hospitalaria y, al año siguiente, se denominó Ministerio de Salud Pública, título con el que aún se conoce.

Con una rica historia de su salud pública, Cuba ha experimentado a partir del año 1959 una transformación de la situación de salud de la población en la que el envejecimiento poblacional, la erradicación o la sensible disminución de la 
incidencia de las enfermedades infecciosas, unido a la relativamente alta prevalencia de enfermedades no transmisibles han generado cambios en los patrones de mortalidad y morbilidad. El desarrollo de la infraestructura de servicios de salud organizada en el Sistema Nacional de Salud Único y con poder resolutivo, dotada de recursos humanos científicamente calificados apegados a la ética, el humanismo y la solidaridad, han sido elementos básicos para el desarrollo y extensión del alcance de las acciones de salud en el país.

La existencia de la Escuela Cubana de Salud Pública, con una manera propia y única de la enseñanza, la investigación y la práctica de la salud pública, que ha tomado de diferentes escuelas de Europa, América Latina, Canadá y los Estados Unidos de América, lo mejor de cada una de ellas mezclándolo con las raíces históricas, sociales y multiculturales de Cuba, es una realidad inobjetable que tiene sus antecedentes en la Escuela de Higienistas Cubanos. Si en esta última existieron personalidades como Finlay, Guiteras, López del Valle, Agramonte y tantos más, en la Escuela Cubana de Salud Pública hoy nos encontramos ya con figuras de la talla de Gustavo Aldereguía Lima, J orge Aldereguía Valdés Brito, Daniel Alonso Menédez, Adolfo Álvarez Blanco, Celestino Álvarez Lajonchere, Juan J. Apolinaire Pennini, Pedro Azcuy Henríquez, Manuel Becerra Troya, Luis Córdova Vargas, Luis M. Cruz Cruz, Mario Escalona Reguera, Carlos Font Pupo, Roberto Hernández Elías, Gustavo Kourí Flores, Sergio Ledo Duarte, Elena López Serrano, Carlos Martínez Reyes, Ramón Martínez Rodríguez, Oscar Mateo de Acosta Fernández, Eduardo B. Ordaz Ducungé, Roberto Pereda Chávez, Conrado del Puerto Quintana, Abelardo Ramírez Márquez, José Rodríguez Abrines, Pedro Rodríguez, Bartolomé Sagaró Hidalgo, Flavia Sánchez Manduley, Arnaldo Tejeiro Fernández, Gabriel Toledo Curbelo, Víctor Victorero de la Fé, Leonardo Julio Wertheim y muchos más que haría interminable la lista de personalidades, donde faltarán algunos, dedicados a la teoría, la docencia, la investigación y la práctica de la salud pública. Hombres y mujeres con virtudes y defectos, con aciertos y desaciertos, como todos los seres humanos, pero siempre abrazados al ideario martiano prefiriendo "la estrella que ilumina y mata", ${ }^{9}$ conscientes de que al decidir dedicar sus vidas a la obra de la salud pública, lo escrito el 13 de Septiembre de 1892 por José Martí al Generalísimo Máximo Gómez podría volver a cobrar vigencia en sus propias existencias: "...Yo ofrezco a Ud., sin temor de negativa, este nuevo trabajo, hoy que no tengo más remuneración que brindarle que el placer del sacrificio y la ingratitud probable de los hombres..."10

Uno de los retos más trascendentes del Sistema Nacional de Salud de Cuba es revitalizar en todos sus aspectos la Escuela Cubana de Salud Pública con énfasis en la consolidación de un sistema académico, de pensamiento y de acción que integre y conforme a las mejores experiencias de la práctica de la salud pública cubana con alcance nacional e internacional de forma tal que surjan los relevos de sus personalidades más relevantes en las nuevas generaciones de sanitaristas cubanos, aspecto este que en los momentos actuales, ya en la segunda década del siglo XXI, no se avizora con claridad en un futuro inmediato y se constituye como una franca debilidad del Sistema.

\section{La necesidad del perfeccionamiento constante del Sistema Nacional de Salud y de la observancia de las circunstancias del contexto social}

El Sistema Nacional de Salud de Cuba ha estado constantemente en perfeccionamiento desde su surgimiento y consolidación.

Los efectos primarios de la profunda crisis económica que enfrentó el país a inicios de la década del 90, y sus efectos secundarios, provocaron el deterioro infraestructural de instalaciones, serias carencias de recursos materiales, deficiencias en el 
desempeño profesional, falta de capacitación gerencial para la conducción del sistema de salud a nivel de las unidades, acompañadas de un incremento de las demandas de atención en hospitales, y la insatisfacción creciente de la población, sobre la atención a urgencias y ambulatoria. ${ }^{11}$

La estructura de los trabajadores de la salud según ocupación en el 2009, muestra que solamente el $5 \%$ de los profesionales dedicados a la Organización y Administración de Salud eran especialistas tal y como se observa en la cita que a continuación aparece:

Los indicadores más elevados eran 119 técnicos medios por 10000 habitantes y 86,5 en personal de enfermería por 10000 habitantes. En ese propio año se contaba con 66,6 médicos por 10000 habitantes, y 10,3 estomatólogos por 10000 habitantes. Al cierre del año 2009, se registraban 74880 médicos en activo, de los cuales el $73 \%$ estaban especializados. El número mayor de médicos se correspondía a los formados en medicina general integral ( $45 \%$ del total), con el $75 \%$ de ellos especialistas, seguido de los dedicados a la organización y administración en salud (aproximadamente el $20 \%$ del total) con solo $5 \%$ de especialistas. Otras de los médicos más representados se dedicaban a la medicina interna, la ginecoobstetricia, y la pediatría. ${ }^{12}$

En el momento actual, el país con su Sistema Nacional de Salud está empeñado en la realización de nuevas transformaciones dentro del mismo.

Deberán esperarse diferencias territoriales en los avances de las transformaciones, ni la calidad de la atención va a ser igual en todos los territorios, ni con los mismos servicios en dos territorios habrá igual satisfacción. Es evidente que en la capital del país, las expectativas, en especial de servicios de alta tecnología, son superiores, comparadas con las de áreas montañosas del oriente cubano. Junto a la administración de salud, otras ciencias como la economía, la geografía, la sociología, la psicología, la antropología y la estadística, tendrán mucho que aportar en el abordaje complejo de los temas de distribución y equidad, tanto en el orden teórico cuanto práctico, no solo en el sector que nos ocupa, sino en el resto de los sectores que integran la salud pública. ${ }^{13}$

Para lograr estas transformaciones no deberían obviarse experiencias pasadas, caminos ya transitados por otras generaciones, circunstancias del contexto histórico social, los escritos de los clásicos de la salud pública en el ámbito nacional e internacional sin que estos se constituyan, por supuesto, como dogmas inmutables al cambio como esencia de la dialéctica. Rojas Ochoa señala:

Yo aspiraría, -no sé si es lo que va a venir o no- a algo ligado estrechamente a la formación del médico que atienda más a la dinámica social de la salud y de la enfermedad. Más promoción de salud...una mayor atención a los problemas sociales que tienen que ver con la salud del hombre... es preciso que el nuevo modelo no le rinda tanto culto a la alta tecnología, que nos asentemos un poco en los viejos procedimientos, tan eficaces, como la clínica. ${ }^{14}$

\section{CONSI DERACI ONES FI NALES}

En la actual fase de desarrollo de la salud pública cubana se impone la necesidad de contar con nuevas generaciones de administradores de salud reconocidos socialmente, competentes, estables, con sentimientos de pertenencia y motivación consecuente hacia la labor que llevan a cabo con absoluta honradez y dedicación, que sean capaces no solamente de conducir los procesos con eficiencia sino 
también con eficacia y con efectividad dentro del ordenamiento institucional existente.

No es posible obviar el hecho de que se ha producido un daño social al no existir durante más de 20 años graduados de la especialidad de Organización y Administración de Salud Pública y con ello no poder contar con especialistas que pueden también, acorde a su formación y entre otras acciones, considerar las influencias socioculturales sobre el proceso salud enfermedad en el diseño de las acciones de salud y del propio sistema.

Los intentos en todo este tiempo de ocupar el espacio vacío dejado por la especialidad por otras modalidades docentes, con cambios frecuentes en su concepción y diseño, no han alcanzado resultados estables en el empeño que puedan traducirse, entre otras cosas, en la sostenibilidad de la capacidad de dirección del Sistema Nacional de Salud y su propia coherencia.

De manera que cada día se hace más patente la necesidad de formar y contar con nuevos profesionales de la salud pública egresados del subsistema de formación de posgrado altamente calificados, con sólidos y amplios conocimientos y habilidades, capaces de proyectar las acciones de salud a la población, para lo cual deben poseer una clara concepción teórico-práctica acerca de los problemas de la salud y la enfermedad y de la influencia sobre ella de los factores sociales, psicológicos, biológicos, químicos y físicos en la constante interacción del hombre con el medio. Unido a lo anterior, deberán ser capaces de poseer una sólida preparación política, humanista, solidaria, ética, y elevada formación científico técnica que les permita el análisis de problemas que afectan la salud de la población y dominar métodos, técnicas y procedimientos que propicien el perfeccionamiento constante del sistema nacional de salud y el mejoramiento del estado de salud de la población.

Pero todo ello no se logra por arte de magia ni por un rosario de buenas intenciones enunciadas con la mejor voluntad del mundo en numerosos documentos.

Tomemos también, en el empeño de no perder la confianza en que todo tiempo futuro tiene que ser mejor, las palabras de José Martí cuando sentenció: "Tengo fe en el mejoramiento humano, la vida futura, la utilidad de la virtud..." ${ }^{15}$

Cabe enfatizar, como colofón, que resulta muy difícil ignorar

[...] la existencia de una especialidad, que ha surgido en la práctica y se ha desarrollado desde hace más de 200 años, que se ha llamado de diversos modos, que ha sido y es el quehacer de innumerables profesionales que en diversas épocas y circunstancias han intentado dirigir sobre bases científicas las tareas de la protección de la salud de la población. ${ }^{16}$

Disponer de profesionales de la salud pública competentes es una prioridad absoluta para el buen desempeño y fortalecimiento constante del Sistema Nacional de Salud. En la consecución de este noble propósito, la especialidad de Organización y Administración de Salud Pública, una vez que surja de nuevo con este nombre o con otro, se consolide en el tiempo y cumpla su encargo social coherentemente, podrá ser de suma utilidad.

Confiemos en que más temprano que tarde aparezca de nuevo la especialidad para bien del pueblo cubano y de la histórica y necesaria Escuela Cubana de Salud Pública. 


\section{REFERENCI AS BI BLI OGRÁFICAS}

1. Vidal Ledo MJ . La Salud Pública: Sistema complejo. Revista Infodir [Internet]. 2011[ citado 23 Mar 2012]; 13. Disponible en: http://bvs.sld.cu/revistas/infd/n1311/infd0513.htm

2. Mannheim K. Das Problem der Generationen. In: Karl Mannheim. Wissenssoziologie. Auswahl aus dem Werk. Hg. von Kurt H. Wolff, Luchterhand, Neuwied/Berlín 1964.

3. Santamaría R. Los desafíos del Posgrado en América Latina. México: UDUAL; 1995.

4. Ministerio de Salud Pública. Resolución Ministerial 142. Plan de acción para incrementar la calidad de los recursos humanos en el Sistema Nacional de Salud. La Habana: MINSAP; 1996.

5. Santovenia Echaine ES. El protomedicato de La Habana. Cuad Hist Sanit, 1952.

6. Delgado García G. Desarrollo histórico de la salud pública en Cuba. Rev Cubana Salud Pública [Internet]. 1998 [citado 29 Abr 2012 ];24(2):110-8. Disponible en: http://scielo.sld.cu/scielo.php?script=sci arttext\&pid=S086434661998000200007\&lng=es

7. Rodríguez Expósito C. Dr. Juan Guiteras. La Habana: Editorial Cubanacán; 1947.

8. La primera Secretaría de Sanidad del mundo se creó en Cuba. Cuad Hist Salud Pública. La Habana, 1964.

9. Martí J. Prologo. Versos Libre. Poema "Yugo y Estrella" [Internet]. 1882[citado 23 Mar 2012]. Disponible en:

http://www.edu.mec.gub.uy/biblioteca_digital/libros/M / Marti, \% 20] ose\% 20$\%$ 20Versos\%20libres.pdf

10. Martí J. Carta al General Máximo Gómez. En: Consejo Nacional de Cultura, Consejo Nacional de Universidades, editores. Obras Completas. Cuba Política y Revolución. T2. La Habana: Editorial Nacional de Cuba; 1963. p. 162-3.

11. Chang M, Alemán ML, Cañizares MP, I barra AM. Satisfacción de los pacientes con la atención médica. Rev Cubana Med Gen Integr [Internet] 1999[citado 23 Mar 2012]; 15 (5): 541-7. Disponible en:

http://scielo.sld.cu/scielo.php?script=sci arttext\&pid=S0864-

21251999000500009\&lng=es\&nrm=iso\&ting=es

12. Ministerio de Salud Pública. Anuario Estadístico de Salud 2009. La Habana: MI NSAP; 2010.

13. Iñiguez Rojas L. Aproximación a la evolución de los cambios en los servicios de salud en Cuba. Rev Cubana Salud Pública [Internet]. 2012[citado 23 Mar 2012]; 38 (1). Disponible en: http://scielo.sld.cu/scielo.php?script=sci_arttext\&pid=S086434662012000100011\&lng=es\&nrm=iso\&tIng=es 
14. Rodríguez Oliva LI. Modelos de salud en Cuba. Habla un protagonista. Entrevista a Francisco Rojas Ochoa. Revista Temas. 2006; (47): 35-43.

15. Martí ]. Prologo. Ismaelillo [Internet]. 1882[citado 23 Mar 2012]. Disponible en: http://josemarti.org/jose_marti/obras/poesia/ismaelillo/01ismaelillointro.htm

16. Rojas Ochoa F. Fundamentación de la especialidad de Salud Pública. Rev Cubana Salud Pública [Internet]. 2012 [citado 23 Mar 2012]; 37(supl. 5). Disponible en: http://bvs.sld.cu/revistas/spu/vol37_05_11/spu09511.htm

Recibido: 19 de febrero de 2012.

Aprobado: 4 de abril de 2012.

Luis Suárez Rosas. Escuela Nacional de Salud Pública. Calle 100 No. 1132 e/ E y Perla. Altahabana, Municipio Boyeros. La Habana, Cuba. Correo electrónico:

Isuarez@infomed.sld.cu 